\title{
A influência da atividade física para pessoas com obesidade: uma revisão integrativa
}

\author{
The influence of physical activity for people with obesity: an integrative review \\ La influencia de la actividad física para las personas con obesidad: una revision in integrativa
}

Recebido: 21/12/2021 | Revisado: 28/12/2021 | Aceito: 05/01/2022 | Publicado: 14/01/2022

\author{
Jessica de Almeida Alves \\ ORCID: https://orcid.org/0000-0001-6215-1582 \\ Universidade Estadual da Paraíba, Brasil \\ E-mail: jessicaalmeida215@outlook.com \\ Karina Araujo Andrade \\ ORCID: https://orcid.org/0000-0002-9753-3772 \\ Universidade Estadual da Paraíba, Brasil \\ E-mail: karinandrrade@gmail.com \\ Clésia Oliveira Pachú \\ ORCID: https://orcid.org/0000-0002-7356-6297 \\ Universidade Estadual da Paraíba, Brasil \\ E-mail: circularsaude.uepb@gmail.com
}

\begin{abstract}
Resumo
Objetivo: O presente estudo objetivou investigar a influência da atividade física para pessoas com obesidade por intermédio de uma revisão integrativa. Metodologia: A presente pesquisa acerca da influência da atividade física na obesidade foi realizada por meio de uma revisão integrativa nas bases de dados SciELO, DOAJ e RBONE, utilizando o termo "obesidade AND atividade física". Resultados: Foi possível verificar que as principais causas da incidência de obesidade estão relacionadas aos hábitos sedentários que a humanidade vem adquirindo. Possivelmente, influenciados pelo abuso dos meios tecnológicos de comunicação, aliado às demais desordens de caráter biopsicossociais características da sociedade em que estamos inseridos. Nesse sentido, predisposto a afetar todos os públicos, independentemente de suas idades e/ou condições socioeconômicas. Conclusão: Fica evidenciado que um bom programa de atividades físicas, quando encaixados no cotidiano, pode fazer toda a diferença neste cenário. Assim, contribuindo de forma excepcional na prevenção e controle da obesidade. Ademais, ficou constatado que a atividade física se apresenta como fator indispensável na prevenção e tratamento da obesidade. Sugere-se a sensibilização de todos os públicos para prática de atividade física e, consequente redução do número de obesos.
\end{abstract}

Palavras-chave: Obesidade; Exercício físico; Qualidade de vida.

\begin{abstract}
Objective: This study aimed to investigate the influence of physical activity on people with obesity through an integrative review. Methodology: This research on the influence of physical activity on obesity was carried out through an integrative review in the SciELO, DOAJ and RBONE databases, using the term "obesity AND physical activity". Results: It was possible to verify that the main causes of obesity incidence are related to sedentary habits that humanity has been acquiring. Possibly influenced by the abuse of technological means of communication, combined with other disorders of a biopsychosocial character characteristic of the society in which we live. In this sense, predisposed to affect all audiences, regardless of their age and/or socioeconomic conditions. Conclusion: It is evident that a good physical activity program, when fitted into daily life, can make all the difference in this scenario. Thus, making an exceptional contribution to the prevention and control of obesity. Furthermore, it was found that physical activity is an essential factor in the prevention and treatment of obesity. It is suggested to raise awareness of all audiences to practice physical activity and, consequently, reduce the number of obese people.
\end{abstract}

Keywords: Obesity; Exercise; Quality of life.

\section{Resumen:}

Objetivo: Este estudio tuvo como objetivo investigar la influencia de la actividad física en personas con obesidad a través de una revisión integradora. Metodología: Esta investigación sobre la influencia de la actividad física en la obesidad se realizó mediante una revisión integradora en las bases de datos SciELO, DOAJ y RBONE, utilizando el término "obesidad Y actividad física". Resultados: Se pudo constatar que las principales causas de incidencia de la obesidad están relacionadas con los hábitos sedentarios que viene adquiriendo la humanidad. Posiblemente influenciado por el abuso de los medios tecnológicos de comunicación, combinado con otros trastornos de carácter biopsicosocial característicos de la sociedad en la que vivimos. En este sentido, predispone a afectar a todos los públicos, independientemente de su edad y / o condiciones socioeconómicas. Conclusión: Es evidente que un buen programa de 
actividad física, cuando se integra en la vida diaria, puede marcar la diferencia en este escenario. De esta forma, aportando una contribución excepcional a la prevención y el control de la obesidad. Además, se encontró que la actividad física es un factor fundamental en la prevención y tratamiento de la obesidad. Se sugiere concienciar a todos los públicos para que practiquen actividad física y, en consecuencia, reducir el número de personas obesas.

Palabras clave: Obesidad; Ejercicio físico; Calidad.

\section{Introdução}

A obesidade, considerada como o acúmulo excessivo de gordura no corpo, resultado de um desequilíbrio crônico entre a energia ingerida e a despendida. Estudos realizados acerca da obesidade familiar tiveram como resultado que os fatores genéticos são importantes na determinação da obesidade. Diante disso, pode-se afirmar que, o indivíduo já pode nascer com a predisposição genética para essa doença crônica. Como fator desencadeante, também, pode-se considerar as variações hormonais que podem ser influenciadas pelo tecido adiposo (Santos, 2010).

A doença crônica obesidade e doenças a ela associadas causam grande impacto ao sistema de saúde pública e aos planos de saúde. Para que se possa intervir na incidência da obesidade e na sua prevalência, entre diversas ações necessárias está o diagnóstico precoce. Este pode ser realizado pelos pais e pela própria escola, em casos de início de obesidade em época escolar. Deve-se ainda, destacar a atenção para alimentação, que nos últimos tempos tem demonstrado o aumento do consumo de alimentos industrializados. É válido ressaltar, o consumo de alimentos industrializados, não só por classe alta, mas por todas as classes sociais, consequentemente havendo uma diminuição do consumo de alimentos in natura (Junior, 2010).

De certo modo, segundo o mesmo autor, algo que influencia muito a obesidade é o sedentarismo. Essa ausência de atividade física, comodismo que afeta diversas pessoas, proporcionando a ausência do gasto calórico. O sedentarismo facilita o desencadeamento e manutenção da obesidade, enquanto observado que os exercícios físicos conduzem a diminuição do volume das células adiposas em razão da redução da formação e estímulo da degradação de triglicérides nos depósitos de gordura. Na prática do exercício, de forma fisiológica ocorre com o deslocamento dos ácidos graxos livres e glicose para o tecido muscular. Assim, aumenta a captação e oxidação desses substratos. Observando isso, principalmente em exercícios prolongados, destacando mais de meia hora. Dessa maneira, havendo a diminuição de glicose em paralelo ao aumento da utilização de ácidos graxos.

Nesse contexto, a prática da atividade física representa o componente que proporciona e facilita o gasto energético diário. Sabendo que essa prática impacta no equilíbrio do balanço energético, a atividade física pode ser a barreira que impede o ganho de peso e pode ser utilizada ainda para o tratamento da obesidade. Assim, sabendo que a atividade física realizada regularmente irá trazer inúmeros benefícios, podendo ter impacto na redução do número da taxa de obesidade, proporcionando assim, um maior controle no ganho de peso (Vianna et al., 2008). Diante disso, considera-se que a obesidade representa grande preocupação por todas as suas repercussões sistêmicas, proporcionando um desequilíbrio crônico.

Pergunta-se: como a prática de atividade influencia o tratamento da obesidade? O presente estudo objetivou investigar a influência da atividade física para pessoas com obesidade por intermédio de uma revisão integrativa.

\section{Metodologia}

Neste artigo, foi utilizado como metodologia de pesquisa para investigar a influência que a atividade física possui sobre a obesidade, uma revisão integrativa realizada a partir das bases de dados Scielo, DOAJ e Rbone (Figura 1), conduzida no período de março de 2020, com palavras-chave acrescida de booleano, "obesidade” AND “exercicio físico". 


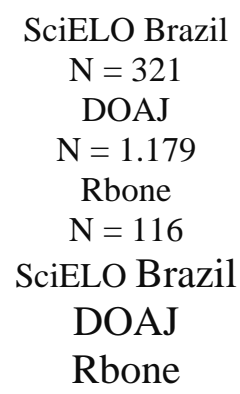

Figura 1 - Dados coletados.

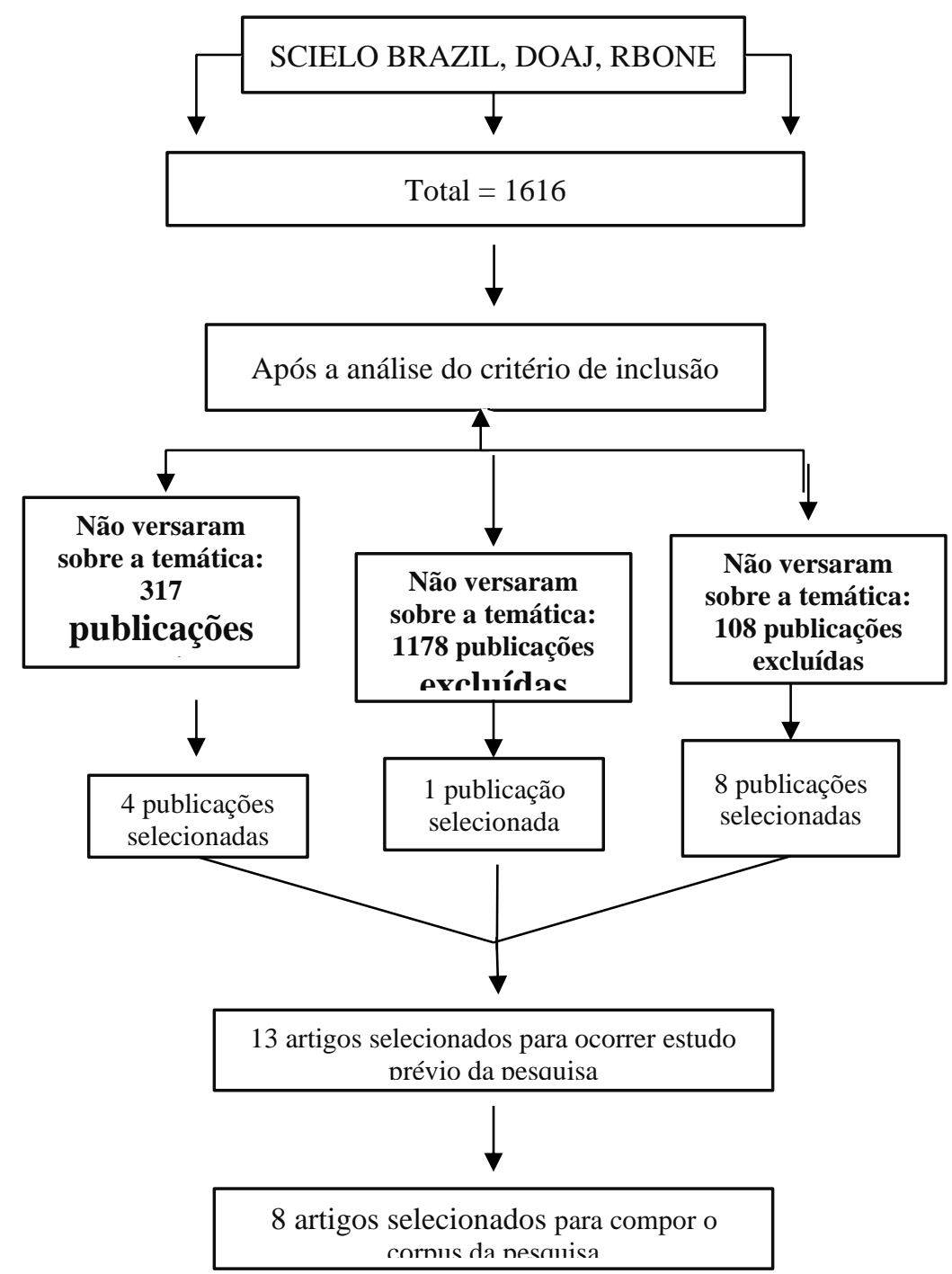

Fonte: Elaborada pelos autores (2021).

Assim, focando no conceito das vastas possibilidades de movimentação que o termo "atividade física" possui, sem intenção de restrição ao exercício físico e seu controle de variáveis. Foi utilizada como fonte de dados para o desenvolvimento deste estudo, uma revisão integrativa a partir da coleta de dados de artigos acadêmicos já publicados, utilizando como meio de busca publicações na língua portuguesa encontradas pelo uso do termo: "obesidade AND atividade física". A análise possui 
como critérios de inclusão apenas materiais produzidos entre os anos de 2000 e 2021, com foco exclusivo na língua portuguesa e no território nacional. Abordando a relação da atividade física sobre a doença crônica obesidade, sem grupo especial.

\section{Resultados e Discussão}

De acordo com os artigos analisados durante essa pesquisa, foi verificado o quanto a presença de atividade física se encontra escassa no cotidiano das pessoas, quando observada de um ponto de vista mais amplo (Tabela 1).

Tabela 1 -

\begin{tabular}{llll}
\hline Ano & Autores & Revista & Tipo de estudo \\
\hline 2011 & CAVALCANTE, et al. & $\begin{array}{l}\text { ABRASCO - Associação Brasileira de } \\
\text { Saúde Coletiva }\end{array}$ & Estudo exploratório qualitativo \\
\hline 2012 & MACIEL, et al. & Revista de Nutrição & Pesquisa de campo qualitativa \\
\hline 2014 & FILHO, et al. & $\begin{array}{l}\text { Revista Brasieira de Obesidade, } \\
\text { Nutrição e Emagrecimento }\end{array}$ & Pesquisa de campo qualitativa \\
\hline 2017 & NEVES; SOUZA; FUGISAWA & $\begin{array}{l}\text { Revista Brasileira de Medicina do } \\
\text { Esporte }\end{array}$ & Estudo transversal qualitativo \\
\hline TOTAL & - & - & 4 \\
\hline
\end{tabular}

Fonte: Elaborada pelos autores (2021).

O que torna a probabilidade de incidência de sobrepeso e obesidade cada vez maior. Prejudicando a natureza da qualidade de vida do ser humano em todos os seus aspectos. Embora muito se fale atualmente sobre hábitos saudáveis, novas técnicas de emagrecimento e diferentes modalidades esportivas, situar-se em uma sociedade cada vez mais tecnológica vem alterando drasticamente a rotina diária dos brasileiros, e habituando as crianças a reproduzirem um estilo de vida cada vez mais sedentário. Evidenciando a fala da autora Cavalcanti et al. (2011):

Entre a consciência da necessidade da mudança de comportamento e sua real efetivação, estabelece-se uma longa distância. Mudar o comportamento e alterar hábitos arraigados prejudiciais à saúde e à qualidade de vida não se fazem por mágica ou pela simples vontade de mudar; é preciso ter disciplina, persistência, determinação, e incluem também o equilíbrio entre os estados físico, funcional, psicológico, social e os fatores econômicos. (Cavalcanti et al, 2011).

Cavalcanti et al. (2011), em sua pesquisa de caráter qualitativo, realizou uma intervenção nutricional associada à atividade física em dezoito idosas, com idades entre 60 e 76 anos da cidade de João Pessoa- PB, onde, nove possuíam sobrepeso e nove apresentavam obesidade. Obtendo através da aplicação de um programa de ginástica e dieta hipocalórica, entrevistas acerca das experiências das assistidas, podendo constatar através dos depoimentos das participantes ao final, maior disposição para a execução de suas atividades diárias, perda de peso considerável, que repercurtiu em uma sensação de leveza e bem estar físico e emocional, trazendo mais autoestima e autoconhecimento, além de uma mudança acentuada no estilo de vida, efetivada devido ao cuidado com a alimentação e a prática de ginástica que foi claramente reconhecida pelas participantes. Permitindo a Cavalcanti et al. (2011) construir a idéia de que a dieta prescrita e a prática de atividade física quando alinhadas podem retardar sintomas provocados pelo processo de envelhecimento, proporcionando maior vitalidade em todas as áreas e possibilitando a realização das atividades que costumam ser impedidas pela obesidade nesta perspectiva. Afirmando também, que a associação entre corpo e mente, do ponto de vista profissional, se faz necessário para um tratamento de qualidade. O que torna indiscutível a importância da inclusão de um bom planejamento de atividades físicas para um processo de emagrecimento saudável.

Em outro estudo, Filho et al. (2014), avaliou e comparou o nível de atividade física diária e hábitos alimentares de 53 crianças, sendo 33 da escola particular e 20 da escola pública, com faixa etária de 10 a 13 anos de idade, da cidade de Leopoldina- 
MG. Onde foram medidas e registradas inicialmente informações sobre peso, altura, IMC e percentis, e, posteriormente, feita a aplicação de questionários acerca do nível de atividade física e da freqüência alimentar de cada aluno.Comprovando que tanto a escola pública quanto a particular tem preferência por alimentos de menor valor nutricional, onde, na particular ainda assim é maior o nível de conhecimento sobre os macronutrientes. Ambas as escolas se mostraram sedentárias quanto a prática de atividade física, sendo a particular muito próxima de ser considerada moderadamente ativa. Relatando-se que as crianças, independente de sua classe socioeconômica estão deixando cada vez mais de lado a pratica de atividade física em detrimento a atividades voltadas ao caráter virtual e tecnológico, em seus momentos de lazer. Dessa forma, Filho et al. (2014), afirma que embora seus valores de IMC e percentis estejam dentro das recomendações sugeridas até o momento da pesquisa, a diminuição das atividades físicas em conjunto a uma alimentação ruim, sugere um aumento de massa gorda e diminuição da massa magra que tornam as crianças propensas a um quadro de obesidade na fase adulta. O que implica dizer que o incentivo, a conscientização e a prática de atividade física regular, se torna imprescindível como fator de prevenção á obesidade ainda na infância.

Com o objetivo de averiguar e comparar o controle postural e a atividade física em crianças eutróficas, com sobrepeso e obesas com oito anos de idade da Rede Municipal de Ensino de Londrina, PR. Neves (2017), elaborou um estudo feito com 346 participantes, para verificar através da aplicação de um questionário, tanto as variáveis citadas acima, quanto a relação entre atividade física e classificação nutricional dessa amostra. Foi constatado assim, alta prevalência de excesso de peso e sedentarismo onde 40,7\% dessas crianças estão acima do peso e 82,9\% são consideradas sedentárias. Quanto a postura, as meninas apresentaram melhor desempenho comparado aos meninos, e, para concluir sua análise, Neves (2017) deixa claro que a atividade física apresentou-se como fator protetor em crianças eutróficas e com sobrepeso. Evidenciando a relevância da prática de atividade física em mais uma peculiaridade, envolvendo em si, distintos tipos de necessidades, quando estas estão também ligadas ao sobrepeso e a obesidade.

Maciel (2012), analisou o consumo alimentar, estado nutricional e nível de atividade física em comunidade universitária brasileira, com 303 voluntários, destes: 130 homens e 173 mulheres, via coleta de peso e altura, além da disponibilização de questionário virtual acerca da frequência alimentar. Destacando em seus resultados a alta prevalência de inadequação alimentar, sobrepeso e obesidade na amostra avaliada. Onde 16,8\% das participantes do sexo feminino apresentam sobrepeso, e nos participantes do sexo masculino esses dados somam 47\% do total de indivíduos, além de nestes estarem também incluídos os únicos graus de obesidade mórbida. Quanto a alimentação, ambos apresentaram baixa ingestão de nutrientes, porém, as mulheres mostraram se alimentar de forma mais adequada.

Não houve correlação significativa entre grupos de alimentos consumidos, índice de massa corporal e nível de atividade física em ambos os sexos. (Maciel, 2012). Sendo frisada a importância de programas educacionais nutritivos como também o estimulo de atividades físicas no ambiente acadêmico como intervenção a esta problemática. Evidenciando os prejuízos que uma rotina com baixo nível de atividade física pode acarretar, e a relevância de sua aplicação em ambientes e situações que também são fora do convencional esperado, como em academias ou clubes esportivos.

Percebe-se diante das constatações sugeridas, o quanto questões ligadas a prática de atividades físicas, na presença ou ausência, em toda vastidão de possibilidades do termo, possuem relação direta com a doença crônica analisada, obesidade. Devido a mudanças de hábitos no cotidiano, provenientes da inserção da tecnologia, entre tantos outros fatores, as pessoas tem evitado cada vez mais movimentar o corpo em detrimento a meios percebidos como mais fáceis, ágeis e práticos de levar a vida. E, essas mudanças afetam a humanidade de forma generalizada, sem muita distinção de faixa etária, sexo, ou condições socioeconômicas. Embora, todos os fatores biopsicossociais sejam influentes durante o tratamento da obesidade. Objetivo esse, que requer muita disciplina e comprometimento, pois, infelizmente reverter esse quadro não possui a mesma facilidade que adentra-lo! Por isso, destaca-se a importância da prevenção, e o quanto ela é capaz de salvar, melhorar e evitar transtornos á nossas vidas. 
Diante dos benefícios do exercício, destacam-se os seus efeitos fisiológicos que podem ser divididos em dois subgrupos: agudos imediatos e agudos tardios. O agudo imediato representa efeitos imediatamente no momento da execução do exercício. Por outro lado, o agudo tardio, os efeitos ocorrem das 24 até 72 horas. Ainda, faz-se importante observar que os efeitos crônicos são resultado da frequência constante na realização de exercícios. Assim, podendo diferenciar o indivíduo que tem rotina de treinos, de um indivíduo que não realiza exercícios. Foi destacado que os exercícios estão relacionados com maior bem-estar e melhor humor, aumentando a motivação e energia para manter um compromisso a longo prazo e evitar recaídas por meio de seu efeito positivo (Santos, 2007).

Um dos fatores mais preocupantes desta problemática, é o quanto as crianças estão habituadas aos maus exemplos dados pela sociedade em que nasceram. Sendo influenciadas por meios de comunicação como a televisão e a internet, pela escola, e até mesmo, pelos pais dentro de suas próprias casas. O que lhes tira o privilégio da conscientização e da escolha, tornando-os meios de reprodução de maus hábitos. Outro público em que o sobrepeso e a obesidade são bastante preocupantes, é o público de idosos. Pois, a obesidade em si, está ligada a inúmeras outras condições sistemáticas, e, esse fato somado as peculiaridades de saúde prováveis de serem adquiridas durante o processo de envelhecimento, e as limitações sociais, e emocionais enfrentadas devido á propensão da solidão característica do avanço da idade, não trazem uma boa perspectiva. Neste meio termo, há os jovens e adultos, onde até se pode pensar que estes vivem em maior estado de equilíbrio, maturidade e, disposição. Porém, este “equilíbrio" esta "maturidade" e está "disposição' sugeridas, muitas vezes se transformam em desleixo, rigidez e autocobranças excessivas quanto a suas vidas estudantis e profissionais, que acabam fazendo com que deixem a sua saúde e bem estar em segundo plano. Por isso, é necessário clareza para todos os públicos citados, para que saiam do modo automático que foram inseridos, e enxerguem com maior amplitude e amor suas vidas e, como de fato preferem vive-las! Onde a alteração de simples hábitos, com a inserção de um planejamento entre as inúmeras possibilidades de atividades físicas existentes, podem mudar totalmente este cenário.

\section{Conclusão}

As evidências apresentadas permitem constatar que a relação entre a atividade física e a obesidade se apresenta como fundamental, tanto em caráter preventivo, quanto sob uma perspectiva de tratamento da comorbidade. Sendo de extrema relevância a sua conscientização e aplicação á todos os públicos, de todos os sexos e idades, independe de demais fatores. Pois, devido a predisposição ao sedentarismo que o abuso dos meios tecnológicos, aliado a demais desordens comportamentais características da sociedade em que estamos inseridos, é perceptível que os riscos de adquirir algum grau de sobrepeso ou obesidade no futuro é um fato considerável. Nota-se então, que um bom planejamento de exercícios ou atividades físicas inclusas no cotidiano podem reduzir consideravelmente esta probabilidade. Podendo propiciar ao praticante diversos benefícios de caráter cognitivo, emocional, social e físico, se tornando assim, fator indispensável na prevenção, controle e combate desta doença crônica que afeta diretamente todos estes aspectos anteriormente citados, no indivíduo. Sendo capaz de regular medidas, equilibrar hábitos, construir e reconstruir sonhos.

Para as crianças, são necessárias mais educação, lucidez e ação, para os jovens e adultos, maiores estímulos e incentivos, pois, provavelmente já sabem em algum lugar de seus interiores onde esses hábitos irregulares podem os levar. E aos idosos, todas as coisas citadas anteriormente, com uma pitada a mais de persistência e empatia ao lidar com o "excesso de vida" e dores que carregam. Para que assim, possamos nos aproximara cada dia, de uma era mais leve e saudável, não só fisicamente, como também emocionalmente e psicologicamente. 


\section{Referências}

Almeida, A. \& Santos, C. (2007). O enfrentamento da síndrome metabólica em indivíduos obesos: A intervenção da atividade física. Revista Brasileira de Obesidade, Nutrição e Emagrecimento, 1(5), 1981-9919

Battista Francisca et al (2021). Metabolic Response to Submaximal and Maximal Exercise in People with Severe Obesity, Prediabetes, and Diabetes. Obesity Facts, 15(4), 415-424. 10.1159/000517589

Bellicha Alice et al (2021). Effect of exercise training on weight loss, body composition changes, and weight maintenance in adults with overweight or obesity: An overview of 12 systematic reviews and 149 studies. Obesity Reviews, 4(4), 3395-5140. 10.1111/obr.13256

Calvacanti Christiane et al. (2011) Programa de intervenção nutricional associado à atividade física: Discurso de idosas obesas. Ciênc. saúde coletiva, 16 (5). https://doi.org/10.1590/S1413-81232011000500007

Carvalho Simone et al. (2021) Os fatores de risco envolvidos na obesidade no adolescente: uma revisão integrativa. Ciência \& Saúde Coletiva, 26 (3). https://doi.org/10.1590/1413-812320212611.3.30852019

Dunker Karin et al (2021) Effects of participation level and physical activity on eating behavior and disordered eating symptoms in the Brazilian version of the New Moves intervention: data from a cluster randomized controlled trial. Sao Paulo Med. J., 139 (3). https://doi.org/10.1590/15163180.2020.0420.R2.04022021

Evans William et al (2021) Effects of exercise training with weight loss on skeletal muscle expression of angiogenic factors in overweight and obese older men. J Appl Physion, 1;131 (1): 56-63. 10.1152/japplphysiol.00084.2021.

Filho Mauro et al. (2014) Nível de atividade física diária e hábitos alimentares em escolares de 10 a 13 anos da cidade de Leopoldina - MG. Revista Brasileira de Obesidade, Nutrição e Emagrecimento, 8 (47), 1981-9919. https://doi.org/10.1152/japplphysiol.00084.2021

Montero López et al. (2021) Determinantes sociais da saúde e prevalência de sobrepeso-obesidade em mulheres urbanas, segundo nível socioeconômico. Hacia promoc. Salud [online], 26(2), 0121-7577. https://doi.org/10.17151/hpsal.2021.26.2.14.

Maciel Erika et al. (2012) Consumo alimentar, estado nutricional e nível de atividade física em comunidade universitária brasileira. Rev. Nutr., 25(6). https://doi.org/10.1590/S1415-52732012000600003

Navid Lorenzo et al (2021) Nutrition, Physical Activity, and New Technology Programs on Obesity Prevention in Primary Education: A Systematic Review. Int J Environ Res Public Health, 18(19), 34639488. 10.3390/ijerph181910187.

Neves Jessica et al. (2017) Controle postural e atividade física em crianças eutróficas, com sobrepeso e obesas. Rev Bras Med Esporte ,23 (03). https://doi.org/10.1590/1517-869220172303157674

Oliveira Fabiano et al (2021) Efeitos de uma abordagem multi-profissional sobre variáveis antropométricas e de perfil alimentar de adolescentes com sobrepeso ou obesidade J. Hum. Growth Dev. [online].31(2), 0104-1282. http://dx.doi.org/10.36311/jhgd.v31.10185.

Oppert Jean et al (2021) Exercise training in the management of overweight and obesity in adults: Synthesis of the evidence and recommendations from the European Association for the Study of Obesity Physical Activity Working Group. Obes Rev, 4(4). 10.1111/obr.13273.

Santos André et al. (2010) Atividade física e dieta como meios preventivos da obesidade infantil. Revista Brasileira de Obesidade, Nutrição e Emagrecimento, 4 (20). Disponível em: http:// www.rbone. com.br/index.php/rbone/article/view/266

Srivastava Shobhit et al. (2021) Interaction of physical activity on the association of obesity-related measures with multimorbidity among older adults: a population-based cross-sectional study in India. BMJ Open, 11(5). 10.1136/bmjopen-2021-050245.

Sumini Karina et al. (2017) Alimentação, risco cardiovascular e nível de atividade física em adolescentes. Revista Brasileira de Obesidade, Nutrição e Emagrecimento, 11 (61). Disponivel em: http://www.rbone.com.br/index.php/rbone/article/view/481

Tomayko Emily et al (2021) Parent Involvement in Diet or Physical Activity Interventions to Treat or Prevent Childhood Obesity: An Umbrella Review. Nutrients, 13(9), 3227. 10.3390/nu13093227.

Vianna, Marcus et al. (2008) A correlação entre o nível de circunferência de cintura e o grau de atividade física. Revista de Educação Física / Journal of Physical Education, 77(142). doi:10.37310/ref.v77i142.493

Vicent Grote et al. (2021) Observation study an inpatient program for musculoskeletal disorders: The effects of gender and physical activity. Medicine (Baltimore),100(43), 34713836, 10.1097/MD.0000000000027594 